\title{
Disseminated Tuberculosis Presenting with Scrofuloderma and Anterior Staphyloma in a Child in Sokoto, Nigeria
}

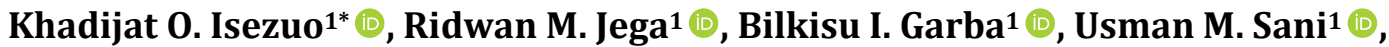 \\ Usman M. Waziri' ${ }^{1}$, Olubusola B. Okwuolise ${ }^{1}$ (), Hassan M. Danzaki² \\ ${ }^{1}$ Department of Paediatrics, Usmanu Danfodiyo University Teaching Hospital, Sokoto, Nigeria \\ ${ }^{2}$ Department of Ophthalmology, Usmanu Danfodiyo University Teaching Hospital, Sokoto, Nigeria \\ Email: *khadisez@yahoo.com
}

How to cite this paper: Isezuo, K.O., Jega, R.M., Garba, B.I., Sani, U.M., Waziri, U.M., Okwuolise, O.B. and Danzaki, H.M. (2020) Disseminated Tuberculosis Presenting with Scrofuloderma and Anterior Staphyloma in a Child in Sokoto, Nigeria. Journal of Tuberculosis Research, 8, 127-135.

https://doi.org/10.4236/jtr.2020.83011

Received: June 1, 2020

Accepted: August 3, 2020

Published: August 6, 2020

Copyright $\odot 2020$ by author(s) and Scientific Research Publishing Inc. This work is licensed under the Creative Commons Attribution International License (CC BY 4.0).

http://creativecommons.org/licenses/by/4.0/

(c) (i) Open Access

\begin{abstract}
Introduction: Disseminated tuberculosis (TB) may occur with skin and ocular involvement which are not common manifestations in children and may lead to debilitating complications. Objective: A child with multi-organ TB involving the lungs, chest abdomen, skin and eyes who had been symptomatic for 3 years is reported. Case Report: A 6-year-old girl presented with recurrent fever, abdominal pain and weight loss of 3 years and skin lesions of a year duration. There was history of pain and redness of the eyes associated with discharge. She was not vaccinated at all. She was chronically ill-looking with bilateral conjunctival hyperaemia, purulent eye discharge with corneal opacity of the right eye. She had significant lymphadenopathy, digital clubbing and ulcerated, discharging swellings around her neck and axilla (scrofuloderma). She had reduced breath sounds and coarse crepitations. Hepatosplenomegaly and ascites were present. She later developed bulging of the upper part of the cornea of the right eye which was diagnosed by the ophthalmologist to be cornea ulcer with anterior staphyloma. Results, Treatment and Outcome: Complete blood count (leucocytosis 12,800 cells), erythrocyte sedimentation rate $(150 \mathrm{~mm} / \mathrm{hr})$ and tuberculin test $(7 \mathrm{~mm}$ induration) were suggestive of TB. Radiological tests were also supportive. She received anti-Kochs in addition to systemic and ocular antibiotics. Skin lesions healed progressively and stopped discharging. Caregivers were counseled on poor visual prognosis of the right eye. She was discharged but lost to follow up. Conclusion: Multi-organ involvement of TB especially ocular and cutaneous TB is not common but is very debilitating. More preventive efforts with vaccination and expertise are needed at lower levels of health care in the community to aid early diagnosis.
\end{abstract}




\section{Keywords}

Scrofuloderma, Staphyloma, Corneal Ulcer, Disseminated TB, Sokoto

\section{Introduction}

Disseminated tuberculosis (TB) is defined as the infection of two or more non-contiguous sites resulting from hematogenous dissemination of Mycobacterium tuberculosis, which occurs as a result of progressive primary infection, reactivation of a latent focus with subsequent spread or rarely through iatrogenic origin [1].

Pulmonary and extrapulmonary tuberculosis despite being one of the oldest diseases in man still continue to be a debilitating scourge especially in developing countries. This is despite an available vaccine to prevent severe forms of the disease. Disseminated tuberculosis (TB) may occur with skin and ocular involvement which are not common manifestations in children and may lead to debilitating organ injuries [2].

Ocular involvement is well described in association with cutaneous tuberculosis; most reports documented are phlyctenular keratoconjunctivitis, tarsitis, and orbital tuberculosis [3]. Extrapulmonary tuberculosis accounts for about 10\% of the total tuberculosis burden. About $1.5 \%$ of these cases have cutaneous disease while ocular involvement in TB is also rare in about $1 \%-2 \%$. These reports are more in adults [4] [5].

We report the case of a 6-year-old girl with multi-organ TB involving the lungs, chest, abdomen, skin and eyes who had been symptomatic for 3 years to highlight the peculiarities of the case and the need for strengthening health care facilities in the community with well trained staff and basic diagnostic facilities.

\section{Case Report}

A 6-year-old girl who presented with recurrent fever, abdominal pain and weight loss of 3 years and skin lesions of a year duration. The skin lesions started as swellings around the neck and axilla which then progressed to become discharging ulcers. There was also history of pain and redness of the eyes associated with discharge of 1 year duration. She was not vaccinated at all. She had contact with an uncle who died after an illness with chronic cough. She had been taken to various health centres in the community before she was referred for tertiary care.

On examination, she was chronically ill-looking, unkempt, and pale. There was bilateral conjunctival hyperaemia, purulent eye discharge with corneal opacity of the right eye. She had significant lymphadenopathy with digital clubbing. There were tender skin lesions around her neck and right axilla characterized 
with necrotic ulceration with undermined edges and discharging sinuses (Figure 1). She was stunted and wasted with height of $83 \%$ and weight of $55 \%$ of expected. Chest examination revealed dull percussion with reduced intensity of breath sounds and coarse crepitations in both lung fields. Abdominal examination revealed hepatosplenomegaly of $8 \mathrm{~cm}$ and $2 \mathrm{~cm}$ respectively. Ascites was demonstrated by shifting dullness.

Central nervous system examination revealed she was conscious but lethargic with no obvious cranial deficit nor signs of meningeal irritation. There was normal muscle tone in all the limbs.

Musculoskeletal examination showed symmetrical wasting, increased prominence of the lower thoracic and lumbar spine with fullness of the right paraspinal area. There was no tenderness.

After 2 days on admission, she was observed to have photophobia with continuous blinking of both eyes. Five days later, she developed bulging of the upper part of the cornea of the right eye.

Ocular findings by the ophthalmologist were that of normal right and left lids with minimal purulent discharge and bilateral corneal scars. The right eye also showed melted cornea with protruded uveal tissue and overlying pseudo-cornea (Figure 2). The left eye pupil was round and reactive with clear lens and red reflex was seen. Assessment was that of right anterior staphyloma and left cornea scar. Visual acuity was limited to perception of light for the right eye. She could count fingers with the left eye. She had medial squint of the left eye.

\section{Investigations Done}

Complete blood count revealed elevated white blood cells of $12.8 \times 10^{3} / \mathrm{L}$ with differential counts of lymphocytes-21.3\%, monocytes-3.2\%, neutrophils-70.2\% and basophils- $0.1 \%$. This suggested an acute bacterial infection probably superimposed on the chronic tuberculous infection. The peripheral blood film showed features of infective process with background hypochromic microcytic anaemia and reactive thrombocytosis.

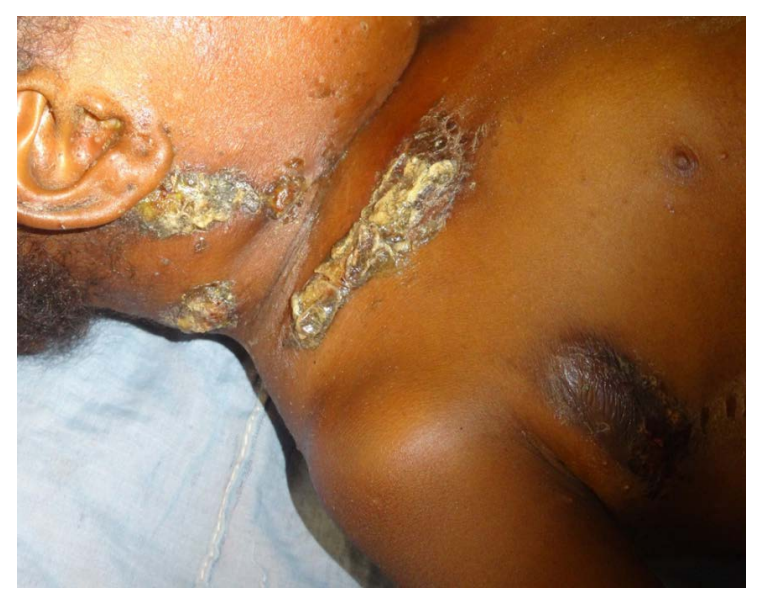

Figure 1. Showing the patient with discharging necrotic ulcers overlying enlarged lymph nodes (scrofuloderma) at presentation. 


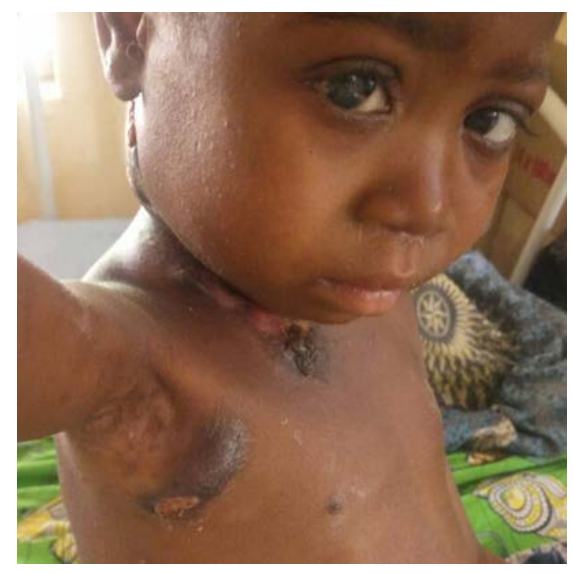

Figure 2. Showing the patient with bulging of the orbital tissues through the cornea (anterior staphyloma) in the right eye, left eye medial squint and scrofuloderma.

The erythrocyte sedimentation rate was also elevated at $150 \mathrm{~mm} / \mathrm{hr}$ in keeping with chronic infection like tuberculosis. The tuberculin skin test zone of induration after 48 hours of tuberculin injection was $7 \mathrm{~mm}$. This was considered positive because of her malnourished status.

Packed cell volume (PCV) was low at $20 \%$. The initial repeat was $22 \%$ and she was placed on haematinics. The repeat PCV before discharge was $28 \%$. Electrolytes, urea and creatinine (E/U/CR) was within normal limits-Na-133 mmol/L, $\mathrm{K}-4.0 \mathrm{mmol} / \mathrm{L}, \mathrm{Cl}-$ not analysed, $\mathrm{HCO}_{3}-26 \mathrm{mmol} / \mathrm{L}$, Urea- $3.3 \mathrm{mmol} / \mathrm{L}$, Creatinine- $0.6 \mathrm{mg} / \mathrm{dL}$.

Swab microscopy of the discharging skin lesions yielded Gram positive cocci in pairs and clusters with growth of Staphylococcus aureus after 24 hours incubation at $37^{\circ} \mathrm{C}$. It was sensitive to Ceftriaxone and Ciprofloxacin.

Gene Xpert test of early morning gastric washings was negative for Acid-Fast bacilli. Retroviral screening (RVS) was negative.

Chest X-ray revealed bilateral patchy para-cardiac and hilar opacities, worse on the right side (Figure 3). The costophrenic angles were within normal limits. The heart was normal in size and contour. Cardiothoracic ratio $=9 / 16.5$. Abdominopelvic ultrasound scan showed excessive bowel gas shadows in the central abdomen with multiple enlarged lymph nodes in the lower abdomen on the right side. One of the largest measured about $21 \times 15 \mathrm{~mm}$. There was mild ascites seen. These findings were suggestive of intraabdominal lymphadenopathy due to abdominal TB.

\section{Treatment}

The working diagnosis on admission was Sepsis complicating disseminated tuberculosis involving the chest (pulmonary TB), skin, lymph nodes (scrofuloderma) and abdomen (tuberculous mesenteric adenitis). She was commenced on intravenous Ceftriaxone and Metronidazole, chloramphenicol eye ointment, chloramphenicol eye drops, multivitamins (Vitamin A and B-complex vitamins, folic acid). She had dressing of the skin lesions with saline and antiseptic solutions. 
After the preliminary investigations supporting tuberculosis, she was commenced on combined anti-TB drugs (isoniazid, rifampicin, pyrazinamide, and ethambutol) and oral steroids.

Patient was co-managed with the Ophthalmologist, Pulmonologist, Nutritionist and Social welfare workers. The caregiver was counselled on the infection (cause, course and treatment) and also concerning the clinical status of the patient and poor visual prognosis of the right eye. Informed consent was also sought and obtained from the caregiver to obtain the clinical photographs of the patient for reporting.

She started responding to treatment with resolution of fever, with progressive reduction and healing of the neck and axillary ulcers (Figure 4 ). She was discharged on request on account of family issues to return after a week. There was no improvement in the vision of the right eye before discharge, nor was there increase in size of the protrusion. Unfortunately, caregivers defaulted follow up since then and their phone number provided during admission documentation could also not be reached.

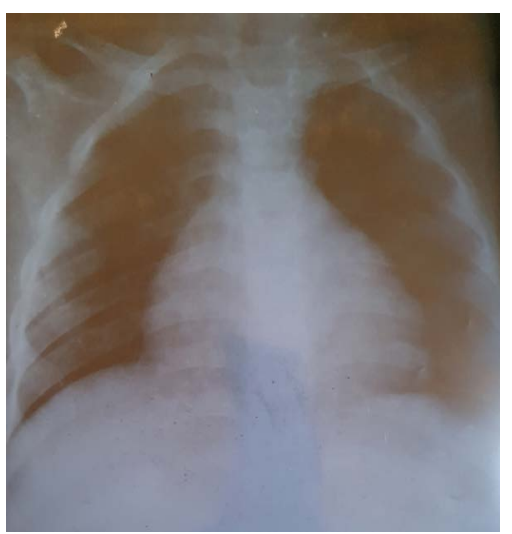

Figure 3. Showing the chest X-ray patchy with patchy opacities more on the right lung zones and hilar area.

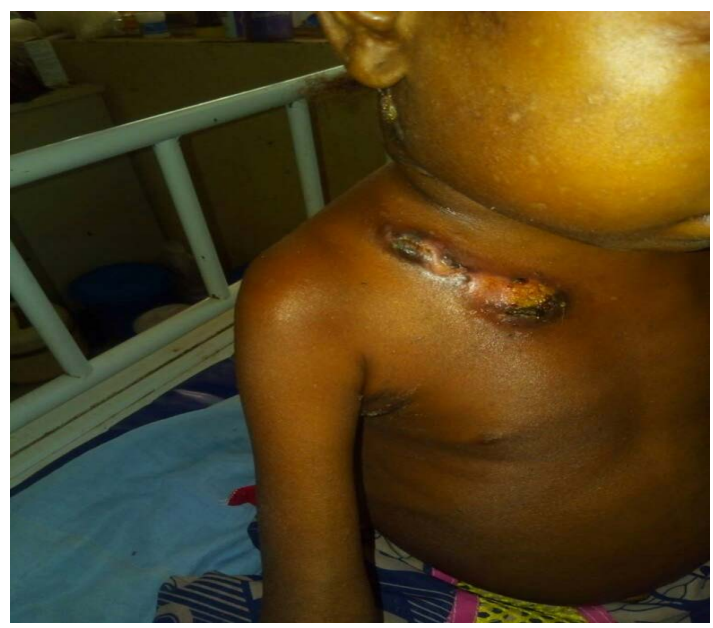

Figure 4. Showing the patient with regressing size of ulcers and lymph nodes (including the pectoral group) after 12 days of antituberculosis drug therapy and wound care. 


\section{Discussion}

Scrofuloderma is the most common form of cutaneous TB among children [6]. Other forms of cutaneous TB include lupus vulgaris, tuberculosis verrucosa cutis, tuberculous chancre, tubercular gumma and other skin lesions called tuberculids. However, scrofuloderma is distinct as it is characterized by asymptomatic swellings that persist for months before softening and ulcerating as seen in our patient. [6] This is as a result of infection and breakdown of the skin overlying a superficial tuberculous focus commonly lymph nodes especially the cervical chain. A study in India links the occurrence of tuberculous cervical lymphadenitis to the ingestion of infected unpasteurized milk [7], a history of consumption of unpasteurized milk was obtained from our patient.

Cutaneous tuberculosis as a direct complication of tuberculous adenitis is recognized presentation of extra-pulmonary TB. However, its occurrence with ocular manifestations in the index patient is rare. The patient had concomitant active pulmonary disease with cutaneous manifestations and bilateral ocular (anterior staphyloma with bilateral corneal ulcers). This posits that direct or indirect immunological reactions triggered by the presence of the mycobacterial antigens may have been responsible for the ocular findings. Such reactions may be driven by tuberculin hypersensitivity in children with active TB [8].

Extrapulmonary TB with concurrent cutaneous and bilateral ocular manifestation with staphyloma is not common in children. In adults, cases of ocular involvement in TB reported include one with isolated corneal melting by Bayraktutar [9] and other cases that had peri-orbital skin and orbital involvement [10] [11]. These manifestations occurred in the absence of active pulmonary involvement. [8] However, our patient had concomitant pulmonary disease with bilateral ocular (anterior staphyloma with bilateral corneal ulcers) and cutaneous manifestations (scrofuloderma) which may have been occasioned by the prolonged duration of symptoms she had without appropriate diagnosis and treatment.

A staphyloma is a protrusion of the uveal tissues of the eye through a weak area on the surface of the cornea or sclera [12]. It usually follows a healed corneal ulcer which creates a weak point in the eyeball. The area of protrusion has a bluish-black hue or colour reflecting the underlying uveal tissues of the eye [4]. The weak spot in the cornea or sclera may be as a result of infection though may also be traumatic or post-surgical [4]. It can progressively increase and cause visual loss which our patient presented with. It accounted for $13.1 \%$ of cases of surgical removal of the eye in southern Nigeria [13]. Kandar et al. [4] reported a similar finding of Staphylococcal conjunctivitis complicated by bilateral staphyloma in a young female adult with cutaneous TB. Thus, a plausible explanation is that in susceptible individuals, the chronic inflammatory process associated with TB may predispose to Staphylococcal and other bacterial eye infections which progress to corneal ulcerations and ensuing staphyloma. Our patient also had features of sepsis with positive culture of Staphylococcus aureus.

In resource constraint settings such as ours, low vitamin A levels are a known 
cause of staphyloma [14]. In such settings, hypovitaminosis A is often associated with disease conditions like measles, fungal infections and malnutrition [15] [16]. Although the vitamin A status was not assessed, fungal cultures were not done, and our index patient did not have measles, her faltered anthropometry suggests she was severely malnourished. Thus, it may be that an underlying hypovitaminosis a either trigged or associated with malnutrition may have increased her susceptibility to disseminated TB and the concomitant occurrence of staphyloma. More so as Qrafli et al. [17] in their case control study demonstrated a likely association between low vitamin A levels and the occurrence of tuberculous disease.

The index patient presented with blurred vision and light sensitivity, these are recognized symptoms of ocular TB. Findings that suggest anterior and posterior uveitis, choroidal tubercles, retinal involvement, keratoconjunctivitis, sclerokeratitis, tuberculous panophthalmitis, ciliary body tuberculoma, choroiditis have all been well described in patients with ocular TB. Of note, is that other protean manifestations like redness of the eye, corneal and scleral inflammation can occur and were seen in our patient [18] [19].

In consonance with this case Bayraktutar and Uçakhan-Gündüz [9] reported finding periorbital swellings and scrofuloderma in their patient. Similarly, spontaneous corneal perforation as seen in this case was also reported by Arora et al. [20].

The definitive diagnosis of ocular TB is established by isolation of $M$ tuberculosis bacilli from ocular tissues. However, because this is difficult to achieve, the diagnosis of ocular TB is often presumed in the presence of suggestive ocular findings in combination with any of the following: systemic findings consistent with TB infection, positive interferon gamma release assay, positive tuberculin skin test in asymptomatic individuals and clinical response to anti-TB treatment further supports a presumed diagnosis of ocular TB [21]. In our patient, the suggestive ocular findings in addition to other systemic findings for TB, with the positive tuberculin test and the rapid response to treatment were used, even though the patient had not completely recovered at the time of discharge and was subsequently lost to follow up.

\section{Conclusion}

Multi-organ involvement of TB especially ocular and cutaneous TB is not common but is very debilitating. More expertise is needed at lower levels of health care in the community to aid early diagnosis to prevent complications as ocular TB which is a preventable cause of blindness and other morbidities. In addition, poverty eradication and strong social support network are needed in the communities to enhance early presentation to hospital and adherence to treatment.

\section{Acknowledgements}

We are grateful to all the staff that were involved in the care of this patient. 


\section{Informed Consent}

This was sought and obtained from the caregiver of the child.

\section{Authors' Contributions}

All the authors have contributed to the final version of this manuscript and have equally contributed to the management of the subject. All have read and approved the final copy.

\section{Conflicts of Interest}

The authors declare no competing interest.

\section{References}

[1] Khan, F.Y., Dosa, K., Fuad, A., Ibrahim, W., Alaini, A., Osman, L., et al. (2016) Disseminated Tuberculosis among Adult Patients Admitted to Hamad General Hospital, Qatar: A Five Year Hospital Based Study. Mycobacterial Diseases, 6, 212. https://doi.org/10.4172/2161-1068.1000212

[2] Singal, A., Aggarwal, P., Pandhi, D. and Rohatgi, J. (2006) Cutaneous Tuberculosis and Phlyctenular Keratoconjunctivitis: A Forgotten Association. Indian Journal of Dermatology, Venereology and Leprology, 72, 290-292. https://doi.org/10.4103/0378-6323.26726

[3] Shakarchi, F.I. (2015) Ocular Tuberculosis: Current Perspectives. Clinical Ophthalmology (Auckland, NZ), 9, 2223-2227. https://doi.org/10.2147/OPTH.S65254

[4] Kandar, A., Sinha, R., Sharma, N., James, K., Bali, S. and Titiyal, J. (2011) Scrofuloderma and Bilateral Anterior Staphyloma of Eye: An Unusual Association. Middle East African Journal of Ophthalmology, 18, 71-73. https://doi.org/10.4103/0974-9233.75894

[5] Donahue, H. (1967) Ophthalmologic Experience in a Tuberculosis Sanatorium. The American Journal of Ophthalmology, 64, 742-748. https://doi.org/10.1016/0002-9394(67)92860-7

[6] Kumar, B. and Kumar, S. (2018) Pediatric Cutaneous Tuberculosis: Indian Scenario. IJPD, 19, 202-211. https://doi.org/10.4103/ijpd.IJPD $63 \quad 18$

[7] Kumar, B., Rai, R., Kaur, I., Sahoo, B., Muralidhar, S., Radotra, B.D., et al. (2001) Childhood Cutaneous Tuberculosis: A Study over 25 Years from Northern India. International Journal of Dermatology, 40, 26-32. https://doi.org/10.1046/j.1365-4362.2001.01165.x

[8] Dalvin, L.A. and Smith, W.M. (2016) Orbital and External Ocular Manifestations of Mycobacterium Tuberculosis: A Review of the Literature. Journal of Clinical Tuberculosis and Other Mycobacterial Diseases, 4, 50-57. https://doi.org/10.1016/j.jctube.2015.11.001

[9] Bayraktutar, B.N. and Uçakhan-Gündüz, Ö. (2015) Ocular Tuberculosis with Progressive Unilateral Corneal Melting. Case Reports in Ophthalmology, 6, 293-297. https://doi.org/10.1159/000439426

[10] Sardana, K., Koranne, R.V., Langan, U., Sharma, R.C. and Bhatnagar, S.K. (2002) Ocular Scrofuloderma with Unilateral Proptosis. The Journal of Dermatology, 29, 232-234. https://doi.org/10.1111/j.1346-8138.2002.tb00255.x

[11] Sharma, S., Juneja, M., Saran, R.K. and Kochhar, A.M. (2006) Bilateral Ocular 
Scrofuloderma with Orbital Tuberculosis. The Indian Journal of Pediatrics, 73, 361-363. https://doi.org/10.1007/BF02825835

[12] Boente, C. (2016) Staphylomas, Congenital, Anterior. In: Schmidt-Erfurth, U. and Kohnen, T., Eds., Encyclopedia of Ophthalmology, Springer, Berlin, 1-2.

[13] Ibanga, A., Asana, U., Nkanga, D., Duke, R., Etim, B. and Oworu, O. (2013) Indications for Eye Removal in Southern Nigeria. International Ophthalmology, 33, 355-360. https://doi.org/10.1007/s10792-012-9700-8

[14] Rabiu, M.M. and Kyari, F. (2002) Vitamin A deficiency in Nigeria. Nigerian Journal of Medicine, 11, 6-8.

[15] Mado, S., Idris, H., Abdullahi, S. and Musa, S. (2018) Anterior Staphyloma in a 6-Month-Old, Severely Malnourished Child Following Measles: A Case Report. Sub-Saharan African Journal of Medicine, 5, 29-31. https://doi.org/10.4103/ssajm.ssajm $23 \quad 17$

[16] Grieser, E.J., Tuli, S.S., Chabi, A., Schultz, S. and Downer, D. (2009) Blueberry Eye: Acquired Total Anterior Staphyloma after a Fungal Corneal Ulcer. Cornea, 28, 231-232. https://doi.org/10.1097/ICO.0b013e318183a3d1

[17] Qrafli, M., El Kari, K., Aguenaou, H., Bourkadi, J.E., Sadki, K. and El Mzibri, M. (2017) Low Plasma Vitamin A Concentration Is Associated with Tuberculosis in Moroccan Population: A Preliminary Case Control Study. BMC Research Notes, 10, Article No. 421. https://doi.org/10.1186/s13104-017-2737-z

[18] Bhatta, S., Thakur, A., Shah, D., Choudhary, M. and Pant, N. (2019) Ocular Manifestations among Systemic Tuberculosis Cases: A Hospital Based Study from Nepal. Journal of Tuberculosis Research, 7, 202-211. https://doi.org/10.4236/jtr.2019.74019

[19] Tabbara, K.F. (2005) Ocular Tuberculosis: Anterior Segment. International Ophthalmology Clinics, 45, 57-69. https://doi.org/10.1097/01.iio.0000155935.60213.ac

[20] Arora, R., Mehta, S., Gupta, R., Gupta, D. and Goyal, J. (2011) Spontaneous Corneal Perforation as the Presenting Feature of Systemic Tuberculosis. British Journal of Ophthalmology, 95, 1176-1177. https://doi.org/10.1136/bjo.2011.204842

[21] Sharma, A., Thapa, B. and Lavaju, P. (2011) Ocular Tuberculosis: An Update. Nepalese Journal of Ophthalmology, 3, 52-67. https://doi.org/10.3126/nepjoph.v3i1.4280 